\section{Fatores ambientais associados à desnutrição infantil em áreas de invasão, Juiz de Fora, MG}

\section{Environmental factors related to child malnutrition in slums, Juiz de Fora, MG}

\author{
Júlio César Teixeira \\ Engenheiro Civil e de Segurança no Trabalho \\ Doutor em Saneamento, Meio Ambiente e Recursos Hídricos \\ Professor Adjunto do Departamento de Hidráulica e Saneamento \\ Universidade Federal de Juiz de Fora \\ Rua Antônio Marinho Saraiva, 125 apto. 202 - Dom Bosco \\ 36.025-555 - Juiz de Fora - MG \\ juliotei@terra.com.br
}

Léo Heller

Engenheiro Civil

Doutor em Epidemiologia

Professor Adjunto do Departamento de Engenharia Sanitária e Ambiental Universidade Federal de Minas Gerais

Trabalho subvencionado pelo Conselho Nacional de Desenvolvimento Científico e Tecnológico (CNPq - Processo $\mathrm{n}^{\circ}$ 476.900/2001-8) e pela Coordenação de Aperfeiçoamento de Pessoal de Nível Superior (CAPES/UFJF - Processo $n^{\circ} 2.573 / 2001-8$ ).

\section{Resumo}

O objetivo deste estudo foi caracterizar a morbidade por desnutrição crônica e por desnutrição aguda, além de identificar os fatores associados a estas doenças, com ênfase para os fatores ambientais, em crianças com idade entre um ano completo e cinco anos completos, residentes em áreas de invasão. Utilizou-se um delineamento transversal de base populacional em áreas de invasão em Juiz de Fora - MG. O critério de inclusão na amostra foi de uma criança por família, por sorteio. A amostra totalizou 659 crianças. A coleta de dados foi feita mediante entrevistas domiciliares. Foram usados modelos de regressão logística para identificar fatores associados às enfermidades. Da amostra, 74 $(11,23 \%)$ crianças apresentaram desnutrição crônica e 40 (6,07\%) apresentaram desnutrição aguda. Os fatores ambientais associados à desnutrição crônica incluíram intermitência no fornecimento de água $(\mathrm{OR}=2,44)$, higiene precária da criança antes da alimentação (OR $=2,05$ ) e lançamento das fezes das fraldas das crianças no peridomicílio $(\mathrm{OR}=2,60)$. Nenhuma variável relacionada ao saneamento e à higiene mostrou-se associada a desnutrição aguda.

Palavras-chave: Desnutrição. Saúde infantil. Fatores ambientais. Saneamento. Higiene. 


\section{Abstract}

The aim of this study was to characterize the morbidity caused by chronic and acute malnutrition and to identify the factors related to such conditions, with special emphasis on environmental factors, in children from one to five years of age, living in areas occupied by homeless or landless people. A population-based cross-sectional study was carried out in areas taken by homeless or landless people in the region of Juiz de Fora, MG. The inclusion criterion for the sample was drawing one child per family. The sample included 659 children. Data were gathered through household interviews. Logistic regression models were used in order to identify disease-related factors. Of the samples analyzed, 74 children (11.23\%) were found to have chronic malnutrition and $40(6.07 \%)$ of them were found to have acute malnutrition. Chronic environmental factors related to malnutrition included: intermittent water supply $(\mathrm{OR}=2.44)$, poor quality child hygiene before feeding $(\mathrm{OR}=2.05)$, and dumping children's diapers with feces in the area around the house $(\mathrm{OR}=2.60)$. No variables connected to sanitation or hygiene were found to be related to acute malnutrition in the study.

Key Words: Malnutrition. Child health. Environmental factors. Environmental Sanitation. Hygiene.

\section{Introdução}

Em 1995, a desnutrição foi responsável por 6,6 milhões das 12,2 milhões de mortes entre crianças menores de cinco anos no mundo. Isto representa $54 \%$ da mortalidade infantil nos países em desenvolvimento. No mesmo ano, mais de 200 milhões de crianças tiveram seu crescimento retardado pela má nutrição. Estas crianças têm maior probabilidade de apresentar baixo desenvolvimento cognitivo, sofrer danos neurológicos, além de menor resistência a doenças. Na idade adulta, podem apresentar maior risco de contrair doenças cardiovasculares, hipertensão, diabetes, altas taxas de colesterol e problemas renais ${ }^{1}$.

A antropometria, pela facilidade de execução e baixo custo, tem-se revelado como o método isolado mais utilizado para o diagnóstico nutricional populacional, sobretudo na infância².

É importante destacar que estudos de coorte têm mostrado serem as medidas antropométricas importantes indicadoras do risco de mortalidade na infância. As crianças que apresentam desnutrição de grau moderado têm o dobro do risco de morrer, enquanto este risco triplica nos casos de desnutrição grave ${ }^{3}$.

A avaliação do estado nutricional das crianças pode ser obtida por meio de estudos transversais, por meio dos índices antropométricos preconizados pela Organização Mundial de Saúde: altura/idade, peso/altura e peso/idade. Assim, a análise do comprometimento do índice altura por idade -o chamado nanismo (stunting) - indica que a criança tem o crescimento comprometido em processo de longa duração e é útil na avaliação da desnutrição crônica. $\mathrm{O}$ déficit no índice peso por altura - o chamado emaciamento (wasting) - reflete um comprometimento mais pronunciado no peso e é útil na avaliação da desnutrição aguda. Já o déficit do peso para a idade (underweight) é um índice isolado, não sendo útil para avaliar a cronologia da perda de peso. Seu déficit pode significar tanto fenômeno recente como antigo ${ }^{4}$. 
Em Juiz de Fora - $\mathrm{MG}^{5}$, no ano 2000, cerca de 78 áreas, alvo de invasões, em processo de legalização ou não, abrigavam aproximadamente 32 mil pessoas em 8,4 mil moradias. Isto significa que pelo menos $7,0 \%$ da população da cidade - 456.796 habitantes ${ }^{6}$ - vivem em situação precária. Dados do Censo de 1991 do $\mathrm{IBGE}^{7}$ mostravam que o número de assentamentos de submoradias no município era 50. Logo, tais fontes indicam que, no período 1991-2000, o número de pessoas em áreas de assentamento subnormal no município, isto é, áreas de invasão, cresceu 3,1 vezes mais do que a população de toda a cidade, respectivamente 5,88\% e 1,89\% ao ano.

Como indicou o Censo 2000, a maioria dos moradores de áreas de habitação subnormal é desempregada, subempregada ou migrante de cidades menores que vêem, em cidades de grande ou médio porte, a chance de uma vida melhor. Assim, frente à falta de emprego e de renda, o problema de ocupação de assentamentos subnormais vem se agravando nos últimos anos, expondo milhões de brasileiros a condições de vida precárias, principalmente em relação à alimentação, à habitação, ao saneamento e às condições de higiene.

\section{Objetivo}

O objetivo do presente estudo foi caracterizar a desnutrição crônica e a desnutrição aguda, identificar os fatores associados a estas doenças, com ênfase nos fatores ambientais, em crianças com idade entre um ano completo e cinco anos incompletos, residentes em áreas de assentamento subnormal áreas de invasão - em Juiz de Fora - MG, visando contribuir para que as intervenções em saúde e saneamento possam ser priorizadas de forma mais eficaz, no sentido de proteger a saúde destas crianças.

\section{Material e Métodos}

Inicialmente, deve-se destacar que o Comitê de Ética em Pesquisas da UFMG COEP, segundo parecer específico datado de 24 de abril de 2002, aprovou o presente estu- do, estando os procedimentos de acordo com os padrões éticos do comitê responsável por experimentos com humanos e de acordo com a Declaração de Helsinki e com a Resolução 196/96 do Conselho Nacional de Saúde.

A seleção das áreas de estudo foi realizada após contato com órgãos da Prefeitura de Juiz de Fora - MG, a saber, a Companhia de Saneamento Municipal S.A. - CESAMA e a Empresa Regional de Habitação de Juiz de Fora S.A. - EMCASA, e organizações não governamentais, como a Pastoral da Criança da Arquidiocese de Juiz de Fora e o Centro de Defesa dos Direitos Humanos CDDH. Considerando as exposições a estudar, 29 áreas de invasão em Juiz de Fora MG, com aproximadamente 2.700 moradias e população estimada em 12.000 habitantes, foram selecionadas, de forma não aleatória, para compor a amostra de estudo.

O método epidemiológico empregado consistiu de um estudo transversal, também denominado de estudo seccional ou de prevalência.

Neste estudo, o dimensionamento do tamanho da amostra foi obtido por meio do software EPI INFO 6.04, a partir de prevalências esperadas para a desnutrição crônica obtidas junto à literatura, uma vez que não foi realizado estudo piloto. Admitiu-se:

- Nível de significância $=5 \%$;

- $\quad$ Poder estatístico do teste $=80 \%$;

- Relação não-expostos/ expostos = 1:1;

- Prevalência esperada entre os expostos $(\text { desnutrição crônica })^{8}=24 \%$.

- Prevalência esperada entre os não-expostos (desnutrição crônica) ${ }^{8}=15 \%$.

Assim, o tamanho da amostra foi fixado em 650 crianças com idade entre um ano completo e cinco anos incompletos residentes em áreas de assentamento subnormal. Foram excluídas as crianças menores de um ano de idade, devido ao seu reduzido contato com o ambiente. É importante destacar que, em cada habitação visitada onde existiam crianças na faixa etária de interesse, uma criança foi sorteada para ser objeto de entrevista, com a verificação do sexo, da idade em meses, da altura e do peso desta criança. 
Considerando a reposição de perdas e as recusas em participar da pesquisa, realizada de maio a julho de 2002, a amostra final totalizou 659 protocolos de entrevista nos 29 assentamentos objetos de estudo. As entrevistas foram realizadas por equipe treinada especificamente para este fim, recrutada entre entrevistadores que trabalharam no Censo 2000 na cidade de Juiz de Fora - MG.

Para obtenção do peso das crianças foram utilizadas balanças portáteis digitais da marca Plenna, modelo MEA-03510, com capacidade de 150 kg e com precisão de \pm 200 g. Já para obtenção da altura das crianças foram utilizados metros dobráveis de madeira com precisão de $\pm 0,1 \mathrm{~cm}$, aferidos, no início dos trabalhos de campo, na Faculdade de Engenharia da Universidade Federal de Juiz de Fora.

Foi considerada doente a criança sorteada com idade entre um ano completo e cinco anos incompletos, residente na área geográfica de estudo, que tenha apresentado escore-z menor que -2 para altura/idade (desnutrição crônica) ou para peso/altura (desnutrição aguda), levando em conta o sexo e a idade em meses desta criança, de acordo com os padrões do National Center for Health Statistics ${ }^{9}$.

Por outro lado, foi considerada não-doente a criança sorteada com idade entre um ano completo e cinco anos incompletos, residente na área geográfica de estudo, que tenha apresentado escore-z maior ou igual a-2 para altura/idade (desnutrição crônica) ou para peso/altura (desnutrição aguda), levando em conta o sexo e a idade em meses desta criança, de acordo com os padrões do National Center for Health Statistics ${ }^{9}$. Este padrão de referência é recomendado pela Organização Mundial de Saúde para crianças com idade entre 0 e 10 anos, tendo sido obtido por meio do estudo de populações eutróficas norte-americanas ${ }^{10}$.

A análise dos dados foi feita por meio do software Statistical Package for the Social Sciences (SPSS), versão 10.0.

A análise com modelo de regressão logística dos dados foi desenvolvida por meio de um processo evolutivo, em etapas seqüenciais, adaptado de Hosmer \& Lemeshow ${ }^{11}$, de forma a permitir a eliminação progressiva das exposições não associadas às doenças. Tal processo envolveu as seguintes atividades:

- Seleção preliminar de variáveis com significância inferior a 0,25 ( $\mathrm{p}<0,25)$ para integrar o modelo;

- Análise das variáveis pré-selecionadas segundo oito subgrupos explicativos;

- Eliminação de variáveis dos subgrupos que apresentaram uma significância superior a $0,15(\mathrm{p}>0,15)$;

- Construção do modelo final com as variáveis remanescentes em cada um dos oito subgrupos, para cada uma das duas doenças estudadas, com a manutenção de variáveis para os modelos finais que apresentaram uma significância inferior a $0,05(\mathrm{p}<0,05)$.

\section{Resultados}

\section{Caracterização do estado nutricional das crianças}

A amostra foi composta por 350 meninos $(53,11 \%)$ e 309 meninas $(46,89 \%)$. Na amostra, 162 crianças tinham idade entre um ano completo e dois anos incompletos $(24,58 \%) ; 154$, idade entre dois anos completos e três anos incompletos (23,37\%); 158 , idade entre três anos completos e quatro anos incompletos (23,98\%); e 185, idade entre quatro anos completos e cinco anos incompletos $(28,07 \%)$.

Da amostra de 659 crianças, 74 (11,23\%) apresentaram escore-z para altura/idade menor do que -2, caracterizando desnutrição crônica, e 40 (6,07\%) apresentaram escore-z para peso/altura menor do que -2 , caracterizando desnutrição aguda. Ainda 70 crianças $(10,62 \%)$ apresentaram escore-z para peso/idade abaixo de -2 , caracterizando déficit ponderal isolado de peso, conforme dados da Tabela 1.

Foi calculada a prevalência padronizada dos três indicadores antropométricos. A curva de freqüência observada para um determinado indicador nutricional antropo- 
Tabela 1 - Prevalência de déficits altura por idade, peso por idade e peso por altura em 29 áreas de invasão de Juiz de Fora, MG, 2002.

Table 1 - Prevalence of height by age, weight by age and weight by height deficits in 29 invaded areas in Juiz de Fora, MG, 2002.

\begin{tabular}{lccccc}
\hline ÍNDICES & \multicolumn{2}{c}{ PREVALÊNCIA PADRONIZADA DO DÉFICIT } & LIMIAR Z-2 & LIMIAR Z-3 \\
& média z & desvio-padrão $z$ & $\begin{array}{c}\text { prevalência } \\
\text { padronizada }(\%)^{(1)}\end{array}$ & $\begin{array}{c}\text { (casos moderados } \\
\text { e graves - \%) }\end{array}$ & (casos graves - \%) \\
\hline Altura/idade & $-0,49$ & 1,41 & 20,16 & 11,23 & 4,10 \\
Peso/idade & $-0,60$ & 1,29 & 22,72 & 10,62 & 2,73 \\
Peso/altura & $-0,31$ & 1,21 & 13,17 & 6,07 & 1,52 \\
\hline
\end{tabular}

${ }^{1}$ A curva de freqüência observada para um determinado indicador nutricional antropométrico diferiu da curva teoricamente esperada em eutróficos em um valor que corresponde à área do afastamento entre estas duas curvas, fornecido nesta tabela em uma cifra percentual - a prevalência padronizada.

${ }^{\prime}$ The frequency curve for a specific nutritional anthropometric indicator differed from the expected curve in eutrophic children at a value that corresponds to the gap between both curves, supplied in the table as a percentage - the standardized prevalence.

métrico difere da curva teoricamente esperada em eutróficos em um valor que corresponde à área do afastamento entre estas duas curvas, normalmente avaliada em valores percentuais-a prevalência padronizada. Em outras palavras, a prevalência padronizada é definida como a cifra global de desnutrição, de forma que o diagnóstico dos casos leves (casos em que o escore-z é menor do que 1,28 e maior ou igual a -2) não seja omitido ${ }^{10}$. Como pode ser observado na Tabela 1, foram encontrados $20,16 \%, 22,72 \%$ e $13,17 \%$, respectivamente, para os indicadores altura/ idade, peso/idade e peso/altura.

\section{Desnutrição crônica}

$\mathrm{Na}$ análise com modelo de regressão logística para desnutrição crônica, 28 variáveis foram incluídas no modelo inicial, sendo: estrutura familiar (11 variáveis), nível socioeconômico (7 variáveis), hábitos higiênicos (2 variáveis), abastecimento de água (2 variáveis), esgotamento sanitário (1 variável), manejo de resíduos sólidos (3 variáveis), drenagem pluvial (2 variáveis) e presença de vetores (nenhuma variável).

No ajuste final do modelo multivariado, cinco variáveis, mostradas na Tabela 2 , apresentaram associação estatisticamente significativa com a desnutrição crônica, sendo uma associada à estrutura familiar (tempo de amamentação); uma ao nível socioeconômico da família (número de cômodos); $\mathrm{e}$ três fatores ambientais (intermitência no abastecimento de água, higiene inadequada da criança antes de se alimentar e lançamento das fezes das fraldas no peridomicílio).

O tempo de amamentação apresentou um odds ratio de 1,92 $(1,04-3,55)$ quando comparado o tempo menor ou igual a três meses com o tempo maior do que três meses. Já o número de cômodos da moradia da criança apresentou um OR de 0,79 (0,66-0,94).

Os três fatores ambientais associados à desnutrição crônica foram: a intermitência no fornecimento deágua da rede pública de abastecimento - quase sempre feita por meio de ligações clandestinas -, por meio da comparação dicotômica "falta água" e "nunca falta água", que apresentou um caráter de risco com OR de 2,44 (1,32-4,4,51); a higiene das mãos da criança antes da alimentação, referente à prática de não lavar ou até lavar com pouca freqüência as mãos da criança antes da alimentação, comparada ao hábito de lavar freqüentemente as mãos da criança comágua ou comágua e sabão, com OR de 2,05 (1,07-3,91); e também foi verificada a existência de risco em relação ao local onde costumam ser lançadas as fezes das fraldas da criança, observando-se que quando se compara o lançamento no terreno, em lotes vagos ou em córregos, com seu lançamento no vaso sanitário ou fossas, ou ainda com as crianças que não utilizam fraldas, encontra-se uma elevada associação de risco com OR igual a 2,60.

\section{Desnutrição aguda}

$\mathrm{Na}$ análise com modelo de regressão logística para desnutrição aguda, 15 variá- 
Tabela 2 - Estimativas, por ponto e por intervalo com 95\% de confiança, dos odds ratio segundo variáveis que permaneceram no modelo de regressão final (Variável resposta: desnutrição crônica) em 29 áreas de invasão em Juiz de Fora, MG, 2002.

Table 2 - Estimates of points and intervals with $95 \%$ confidence intervals, and of odds ratios by variables that remained in the final regression model (response variable: chronic malnutrition) in 29 invaded areas in Juiz de Fora, MG, 2002.

\begin{tabular}{|c|c|}
\hline Variável & OR (IC a 95\%) \\
\hline \multicolumn{2}{|l|}{ Tempo de amamentação* } \\
\hline menor ou igual a três meses & $1,92(1,04-3,55)$ \\
\hline Número de cômodos** & $0,79(0,66-0,94)$ \\
\hline \multicolumn{2}{|l|}{ Intermitência no abastecimento de água*** } \\
\hline Sim & $2,44(1,32-4,51)$ \\
\hline \multicolumn{2}{|l|}{ Higiene antes da criança se alimentar**** } \\
\hline pequena freqüência ou nunca & $2,05(1,07-3,91)$ \\
\hline \multicolumn{2}{|l|}{ Lançamento das fezes das fraldas*****} \\
\hline no próprio terreno, lotes vagos ou córregos & $2,60(1,41-4,80)$ \\
\hline $\begin{array}{l}\text { * Categoria de comparação = maior do que três meses [Comparis } \\
\text { ** Variável contínua [Continuous variable] } \\
\text { *** Categoria de comparação = não [Comparison category =no] } \\
\text { **** Categoria de comparação = quase sempre, com água e sab } \\
\text { soap and water or with water] } \\
\text { ***** Categoria de comparação = no vaso ou a criança não usa fr } \\
\text { wear diapers] } \\
\text { OR = odds ratio }\end{array}$ & $\begin{array}{l}\text { nths] } \\
\text { category = almost always, with } \\
\text { in the toilet or the child does not }\end{array}$ \\
\hline
\end{tabular}

veis foram incluídas no modelo inicial: estrutura familiar (8 variáveis), nível socioeconômico (3 variáveis), hábitos higiênicos (1 variáveis), abastecimento de água (nenhuma variável), esgotamento sanitário (nenhuma variável), manejo de resíduos sólidos (2 variáveis), drenagem pluvial (1 variável) e presença de vetores (nenhuma variável).

No ajuste final do modelo multivariado, as variáveis incluídas na Tabela 3 apresentaram associação estatisticamente significativa com a desnutrição aguda, sendo duas associadas à estrutura familiar (internação no primeiro mês de vida e fornecimento de suplemento nutricional) e uma, ao nível socioeconômico da família (número de cômodos). Nenhum fator ambiental mostrouse associado à desnutrição aguda.

Tabela 3 - Estimativas, por ponto e por intervalo com 95\% de confiança, dos odds ratio segundo variáveis que permaneceram no modelo de regressão final (Variável resposta: desnutrição aguda) em 29 áreas de invasão em Juiz de Fora, MG, 2002

Table 3 - Estimates of points and intervals with $95 \%$ confidence intervals, and of odds ratios by variables that remained in the final regression model (response variable: acute malnutrition) in 29 invaded areas in Juiz de Fora, MG, 2002.

\begin{tabular}{ll}
\hline Exposição & OR (IC a 95\%) \\
\hline Internação no primeiro mês de vida* & \\
$\quad$ Sim & $2,46(1,13-5,37)$ \\
Fornecimento de suplemento nutricional** & \\
$\quad$ Sim & $2,40(1,23-4,66)$ \\
Número de cômodos*** & $0,81(0,67-0,98)$ \\
\hline
\end{tabular}

\footnotetext{
* Categoria de comparação = não [Comparison category =no]

** Categoria de comparação = não [Comparison category $=$ no]

*** Variável contínua [Continuous variable]

$\mathrm{OR}=$ odds ratio
} 
Os três fatores associados à desnutrição aguda foram: a internação no primeiro mês de vida, quando comparada com crianças que não foram internadas neste período, apresentou odds ratio de 2,46 (1,13-5,37); o fornecimento de suplemento nutricional apresentou OR igual a 2,40 (1,23-4,66); e, por último, o número de cômodos que apresentou caráter de proteção com OR de 0,81 $(0,67-0,98)$.

\section{Discussão}

\section{Desnutrição crônica}

Como largamente registrado na literatu$\mathrm{ra}^{3,12}$, o tempo de amamentação menor que quatro meses consiste em um fator de risco para várias doenças, entre elas a desnutrição crônica.

Como já visto, o número de cômodos apresentou um caráter de proteção para a desnutrição crônica. Por um lado, o número de cômodos no modelo representa os indicadores do nível socioeconômico da família da criança. Por outro lado, tal achado sugere que as habitações têm influência na formação de quadros de desnutrição crônica, comprometendo o desenvolvimento das crianças, uma vez que condições inadequadas de espaço, ventilação, iluminação, temperatura do ar e umidade prejudicam o crescimento ${ }^{13}$.

A intermitência no abastecimento de água - caracterizada como fator de risco para a desnutrição crônica - pode se relacionar com o risco à saúde, a partir de três efeitos principais: primeiro, levar a comunidade a consumir água de fontes alternativas de abastecimento, que em função das respostas obtidas na pesquisa, sabe-se serem água de mina, nascente ou poço raso; segundo, pode levar a práticas precárias de higiene das crianças; e, por último, pode implicar contaminação da água de abastecimento na rede de distribuição.

$\mathrm{O}$ abastecimento regular de água, juntamente com o esgotamento sanitário, desempenha um papel fundamental na melhoria do estado de saúde das populações, que não se expressa nos indicadores de mortalidade, mas prejudica a saúde e o estado nutricional dessas populações ${ }^{14}$. No mesmo sentido, sustenta-se que não só a qualidade, mas principalmente a quantidade de água disponível para o consumo das populações, possui um impacto predominante no estado geral de saúde da população, incluindo aí o estado nutricional das crianças ${ }^{15}$.

A importância da higiene das mãos da criança antes da alimentação na prevenção da desnutrição crônica tem sido sistematicamente constatada ${ }^{16}$, embora em outros trabalhos não tenha sido determinada a associação entre higiene das mãos da criança antes da alimentação e a desnutrição crônica ${ }^{17}$.

A caracterização apenas da higiene das mãos da criança antes da alimentação como risco para a desnutrição crônica levanta a hipótese de que tal prática higiênica deve estar representando o nível geral das práticas higiênicas realizadas com a criança pelo responsável. Entretanto, não se pode deixar de destacar o papel protetor das demais práticas de higiene estudadas - preparação da água bebida pela criança, preparação das frutas e verduras utilizadas na alimentação da criança e higiene das mãos da criança após defecar - para a prevenção de inúmeras doenças, como tem sido comprovado por diversos trabalhos ${ }^{14-16}$.

Para a desnutrição crônica foi verificada ainda a existência de risco em relação ao acúmulo das fezes das fraldas no peridomicílio. Tal acúmulo favorece o contato direto das crianças com os excretas, assim como a proliferação de vetores transmissores de doenças que comprometem o desenvolvimento das crianças, contribuindo para o déficit na relação altura por idade, caracterizando quadros de desnutrição crônica.

\section{Desnutrição aguda}

Presume-se que a internação da criança no seu primeiro mês de vida deve-se ao nascimento prematuro ou ao fato de a criança apresentar doenças do período perinatal. A internação da criança no primeiro mês de vida apresentou-se como um fator de risco para a desnutrição aguda. Tal resultado era 
esperado, uma vez que estas crianças apresentam, segundo a literatura especializada ${ }^{3,12}$, quadros de desnutrição aguda que podem perdurar durante anos em famílias de baixa renda.

Surpreendentemente, o fornecimento de suplemento nutricional apresentou-se também como um fator de risco para a desnutrição aguda. Tal afirmação pode em princípio transparecer contraditória, ao se constatar que receber suplemento nutricional no ato da entrevista implica maior risco de apresentar desnutrição aguda. Entretanto, provavelmente não há aqui uma relação de causa e efeito entre exposição e doença. Há, na realidade, uma relação entre doença e sua conseqüência, uma vez que somente as crianças que apresentavam déficit ponderal isolado acentuado - peso/idade - recebiam o suplemento nutricional. Por conseguinte, quando a criança recebia o suplemento nutricional nas áreas estudadas, já apresentava um quadro de desnutrição aguda instalado.

Por último, o número de cômodos da moradia apresentou-se como um fator de proteção para a desnutrição aguda, semelhante ao resultado encontrado para a desnutrição crônica.

Assim, na análise de regressão logística, observou-se que nenhuma variável relacionada ao saneamento e à higiene foi estatisticamente associada à desnutrição aguda, em nível de 5\% de significância, o que é coerente, pois sugere um impacto de longo prazo do saneamento ambiental sobre o status antropométrico $^{16,17}$.

\section{Conclusões}

Considerando a elevada prevalência encontrada para a desnutrição crônica, recomenda-se que os programas voltados para seu controle sejam intensificados nas áreas de assentamento subnormal de Juiz de Fora - MG. Sugere-se atenção especial à saúde de crianças com tempo de amamentação menor ou igual a três meses e/ou que habitam em moradias com menos de quatro cômodos. O estudo indica ainda que, para o controle da desnutrição crônica, são imprescindíveis boas cobertura e qualidade nos serviços de saneamento, em especial abastecimento contínuo de água do sistema público com a eliminação da intermitência no fornecimento. Programas de educação sanitária, visando melhorar as práticas de higiene dispensadas pelo responsável à criança, e o combate ao lançamento das fezes das fraldas da criança no peridomicílio revelam-se imprescindíveis.

Para a prevenção da desnutrição aguda, sugere-se atenção especial à saúde de crianças que foram internadas em seu primeiro mês de vida. Crianças que habitam moradias com menos de quatro cômodos também merecem atenção especial. O estudo indica ainda que nenhuma variável associada ao saneamento e à higiene - abastecimento de água, esgotamento sanitário, resíduos sólidos, drenagem pluvial, hábitos higiênicos e presença de vetores - foi estatisticamente associada à desnutrição aguda, em nível de $5 \%$ de significância.

\section{Referências}

1. Organização Pan-Americana da Saúde (OPAS/OMS). Informativo da Organização Pan-Americana da Saúde sobre desnutrição e má nutrição. Brasília; 2000.

2. Sarni RS. Avaliação da condição nutricional de crianças e adolescentes - método antropométrico. Temas de nutrição em pediatria 2001; 2: 28-38.

3. The United Nations Children's Fund (UNICEF). Situação mundial da infância, 1994. Brasília; 1994.
4. World Health Organization. Use and interpretation of antropometrics indicators of nutricional status. Bulletin of the World Health Organization 1986; 64: 929-41.

5. Prefeitura de Juiz de Fora (PJF). JF Bairros. Juiz de Fora: PJF; 2001.

6. Fundação IBGE. Sinopse preliminar do censo demográfico. Disponível em: <http:// www.ibge.gov.br/ibge/estatistica/universo.php>. Acesso em 03 fev. 2002. 
7. Fundação IBGE. Censo demográfico: resultado do universo relativo às características da população e do domicílio. Rio de Janeiro: IBGE; 1991.

8. Moraes LRS. Impacto na saúde de ações integradas de saneamento ambiental em assentamento humano periurbano de Salvador. Em Heller L, Moraes LRS, Monteiro TC do N, Salles MJ, Almeida LM de, Câncio J. (Org.) Saneamento e saúde em países em desenvolvimento. Rio de Janeiro: CC\&P Editores; 1997.

9. World Health Organization. Measuring change in nutricional status. Genebra; 1983.

10. Goulart EMA. Metodologia e informática na pesquisa médica. Belo Horizonte: EMA Editora; 2000.

11. Hosmer, DW, Lemeshow, S. Applied logistic regression. John Wiley and sons, Nova York; 1989.

12. World Health Organization. Child health and development of the newborn. Genebra; 1992.

13. Heller, L, Möeller, LM. Saneamento e saúde pública. Em Barros, RT de V, Chernicharo, CA de L, Heller, L, von Sperling, M. Manual de saneamento e proteção ambiental para os municípios - volume 2:

Saneamento. Belo Horizonte: Escola de Engenharia da UFMG, 1995.
14. Barreto ML, Teixeira M da G, Strina A. Avaliação epidemiológica do impacto sobre a saúde das ações de saneamento do Projeto Alvorada. Em Projeto Alvorada. Brasília: Governo Federal; 2002.

15. Cairncross S, Kolsky PJ. Comment on: "Water, waste and well-being: a multicountry study". American Journal of Epidemiology 1997; 146: 359-61.

16. Moraes LRS. Health impact of sewerage and drainage in poor urban áreas in Salvador, Brasil [tese de doutorado]. London: London School of Hygiene and Tropical Medicine of University of London; 1996.

17. Azevedo E de A. Exclusão sanitária em Belo Horizonte MG: caracterização e associação com indicadores de saúde [dissertação de mestrado em Saneamento, Meio Ambiente e Recursos Hídricos]. Belo Horizonte: Escola de Engenharia da UFMG; 2003.

recebido em: 21/01/04 versão reformulada apresentada em: 18/06/04 aprovado em: 18/06/04 